This item was submitted to Loughborough's Research Repository by the author.

Items in Figshare are protected by copyright, with all rights reserved, unless otherwise indicated.

\title{
Experimental studies on kinematics and kinetics of walking with an assistive knee brace
}

PLEASE CITE THE PUBLISHED VERSION

http://dx.doi.org/10.1109/ICINFA.2011.5948961

PUBLISHER

(C) IEEE

VERSION

AM (Accepted Manuscript)

\section{PUBLISHER STATEMENT}

This work is made available according to the conditions of the Creative Commons Attribution-NonCommercialNoDerivatives 4.0 International (CC BY-NC-ND 4.0) licence. Full details of this licence are available at: https://creativecommons.org/licenses/by-nc-nd/4.0/

\section{LICENCE}

CC BY-NC-ND 4.0

\section{REPOSITORY RECORD}

Hung, Aaron See-Long, Hongtao Guo, Wei-Hsin Liao, Daniel Tik-Pui Fong, and Kai-Ming Chan. 2019. "Experimental Studies on Kinematics and Kinetics of Walking with an Assistive Knee Brace". figshare. https://hdl.handle.net/2134/21280. 


\title{
Experimental Studies on Kinematics and Kinetics of Walking with an Assistive Knee Brace
}

\author{
Aaron See-Long Hung ${ }^{1}$, Hongtao Guo ${ }^{2}$, Wei-Hsin Liao $^{2}$, Daniel Tik-Pui Fong ${ }^{1}$, and Kai-Ming Chan ${ }^{1}$ \\ ${ }^{1}$ Sport Performance and Biomechanics Laboratory \\ Department of Orthopaedics and Traumatology \\ Prince of Wales Hospital, Faculty of Medicine \\ The Chinese University of Hong Kong \\ Shatin, N.T., Hong Kong, China \\ \{ahung \& dfong\}@ort.cuhk.edu.hk, kaimingchan@cuhk.edu.hk \\ ${ }^{2}$ Smart Materials and Structures Laboratory \\ Department of Mechanical and Automation Engineering \\ The Chinese University of Hong Kong \\ Shatin, N.T., Hong Kong, China \\ \{htguo \&whliao\}@mae.cuhk.edu.hk
}

\begin{abstract}
Assistive knee brace is a species of wearable lower extremity exoskeletons. Such assistive equipment can enhance people's strength and provide desired locomotion to have advantages over wheelchairs, which are commonly used for patients with mobility disorders. However, the integration between the assistive knee brace and the user is challenging as inaccurate alignments may adversely affect the biomechanics of the knee joint. The goal of this study is to evaluate the changes between normal walking and walking with an assistive knee brace in "off" mode. The assistive knee brace was developed by integrating a multifunctional actuator with a custom-made kneeankle-foot orthosis in order to minimize excessive shifting and to improve alignment to the knee joint. Spatial and temporal gait parameters, joint kinematics and joint kinetics parameters were compared. In general, the observed results showed that most of the gait parameters were not affected when walking with the knee brace. The only significant differences were found in knee flexion and knee rotational motions. These results indicated that walking with the developed knee brace provided minimal hindrance to the user and assured that assistive torque can be applied to the knee joint.
\end{abstract}

Index Terms - Assistive knee brace, magnetorheological fluids, multifunctional actuator, gait analysis

\section{INTRODUCTION}

Assistive knee braces are a kind of wearable lower extremity exoskeletons that can enhance people's strength and provide desired locomotion. It is possible to use knee braces to assist elderly or disabled people on improving their mobility in order to solve many daily life problems, such as going up and down stairs and crossing over obstacles. With a continually aging world population, devices that help elderly with mobility problems are in great need. By using assistive knee braces, patients may avoid being bedridden and will be able to maintain their physical activities. They will be able to benefit from the positive effects of exercise and enjoy an active lifestyle.

Studies have been conducted in the development of exoskeletons, such as the RoboKnee developed by Pratt et al [1], the hybrid assistive limb developed by Kawamoto and Sankai [2], and the wearable walking helper by Hirata et al [3]. The commonly used actuation devices in assistive knee braces are electric motors. For such actuators, the brake function would consume much power to maintain posture and might cause safety problems. Some researchers adopted smart fluids in actuation mechanisms. For instance, a rehabilitative knee orthosis equipped electro-rheological (ER) fluids based actuators into knee braces [4]. An orthopaedic active knee brace using magneto-rheological (MR) fluids based shear damper was developed to make the knee brace have a controllable resistance [5]. The developed knee braces using smart fluids could provide controllable torque as assistive devices in passive and semi-active means while only little power is consumed. Furthermore, according to clinical gait analysis (CGA), it can also be found that the knee joint is usually dissipating power during walking [6]. Hence, knee joint dynamics could be matched by a controlled energy dissipative device. However, in some situations for the wearer going upstairs or stepping over obstacles, such knee braces would not be able to help in active ways.

To combine the advantages of electric motors and smart fluids, Chen and Liao developed an assistive knee brace by integrating an MR actuator with electric motor [7]. The MR actuator could function as brake when adjustable torque is preferred; or work as clutch to transfer torque from motor to the brace. With adaptive control, the actuation system worked well and could provide desired torque with better safety and energy efficiency. However, the actuator seemed a bit bulky to be used on human body. A more compact actuator is desired for assistive knee braces.

With this motivation, a multifunctional actuator integrating motor, clutch and brake functions into a single device was designed. Guo and Liao developed two kinds of multifunctional actuators with inner armatures and input/output plates $[8,9]$. The assistive knee brace using the multifunctional actuator combines the advantages of electric motor and MR fluids into one single device, and it has the advantages of less power consumption, improved safety and better controllability.

However, the knee braces only provide assistive torque in single degree of freedom (DOF). Since the human knee has three planes of motion (flexion/extension, valgus/varus, internal/external rotation), it makes the integration between the assistive knee brace and the user very challenging. In fact, it has been shown that inaccuracies exist between angles measured by orthosis compared to angles measured by motion analysis systems [10]. If there are misalignments between the assistive device and the user, it may adversely affect the 
biomechanics of the knee joint and may ultimately injure the user. Therefore, before assistive torque is provided to the user, an evaluation must firstly be conducted to ensure that the knee brace causes no hindrance to the user's normal movement patterns.

The purpose of this study is to evaluate the kinematic and kinetic changes of walking while wearing the assistive knee brace in the "off" mode. Temporal and spatial gait parameters, joint kinematics, and joint kinetic parameters during walking with the developed knee brace will be compared with normal walking. This evaluation will be used to identify possible areas for further improvement in the development of the assistive knee braces.

\section{EXPERIMENTS}

\section{A. Subject}

One healthy male (weight $60.0 \mathrm{~kg}$, height $175.0 \mathrm{~cm}$ ) participated in this experiment. The subject reported no previous lower limb injuries that might affect his gait.

\section{B. Assistive Knee Brace with Multifunctional Actuator}

The assistive knee brace consists of a custom-made kneeankle-foot orthosis (KAFO), a multifunctional actuator (Fig. 1) using MR fluid at the lateral side of the knee, and a hinge joint at the medial side of the knee. The specifications of the KAFO are given in Table I. The KAFO was fabricated by a certified prosthetist-orthotist and was fitted according to the subject's anthropometric measurements. A custom-made KAFO (Fig. 2) was chosen since previous versions of the knee brace demonstrated misalignments to the knee joint and excessive shifting against the leg during walking. The KAFO provided a much better fit to the user and thus was able to significantly reduce the inertia of the assistive knee brace. Additional modifications were also made to the KAFO. Firstly, the KAFO was fabricated using polyethylene due to its durability and
TABLE I

SPECIFICATIONS OF THE KNEE BRACE

\begin{tabular}{|l|l|}
\hline Material & \\
\hline Upper and lower brace & Polyethylene \\
\hline Medial and lateral shaft & Aluminum \\
\hline MR fluids & MRF-132DG \\
\hline Weight & $2.5 \mathrm{~kg}$ \\
\hline Height & $76.5 \mathrm{~cm}$ \\
\hline Maximum width at knee joint & $63.5 \mathrm{~cm}$ \\
\hline Thigh segment diameter & $58.6 \mathrm{~cm}$ \\
\hline Calf segment diameter & $40.0 \mathrm{~cm}$ \\
\hline Foot segment length & $14.8 \mathrm{~cm}$ \\
\hline
\end{tabular}

relatively low cost. Secondly, the length of the foot segment was reduced so that it allows the user to apply a greater forefoot force during push-off with the ankle plantar flexors. Finally, the width of the KAFO at the ankle joint was reduced to increase its flexibility so that the user can have a full range of motion at the ankle joint.

The multifunctional actuator was installed into the KAFO and its joint axis was aligned to the joint axis of the subject's knee joint. During the alignment procedure, the subject was asked to maintain the knee flexed at 30 degrees. At this position, the tip of the patella was identified which corresponded to the joint line between the femur and the tibia. A perpendicular line was then drawn from the tip of the patella to the mid-line of the sagittal knee. Using these points as reference, the center of the knee joint was identified and the MR actuator was attached to the KAFO accordingly (Fig. 3). Finally, a hinge joint was also installed to the medial side of the KAFO using the same procedure. This hinge joint serves to further increase the stability and performance of the assistive knee brace.

The multifunctional actuator is a novel actuator to integrate the advantages of electric motor and MR fluids while decreasing the dimension. The actuator is comprised of two

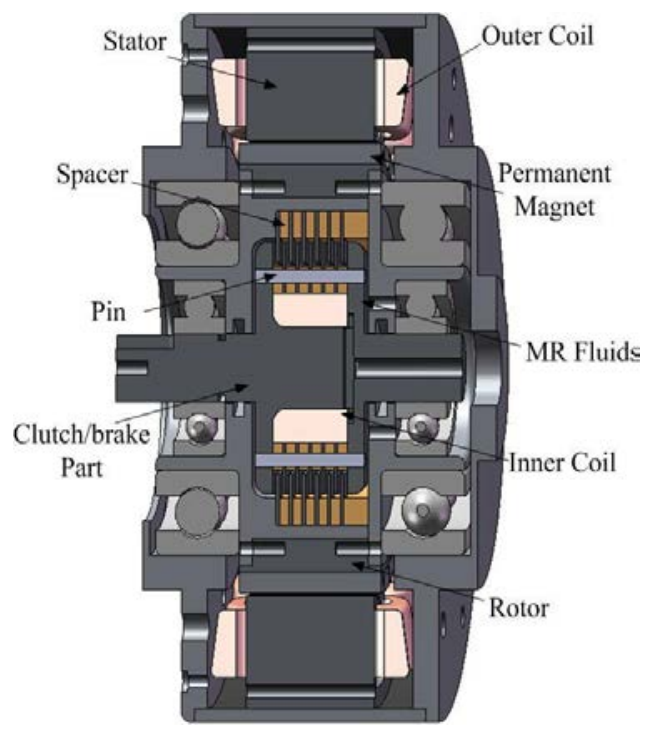

Fig. 1 Schematic of the multifunctional actuator.
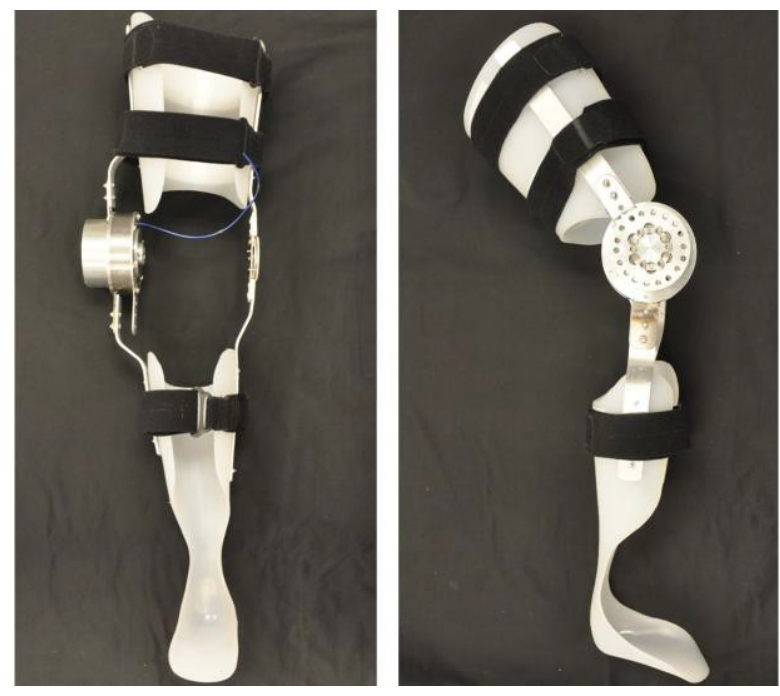

Fig. 2 Knee-ankle-foot orthosis with actuator. 
main parts into a single device: the motor part and the clutch/brake part; each part is associated with the corresponding coils. MR fluids are filled inside the motor part along with the inner clutch/brake part. The motor part converts electric power into mechanical power to provide active torque. With the use of MR fluids, the clutch/brake part could transfer the torque generated from the motor part to outside as a clutch or provide controllable semi-active torque as a brake with less power consumption than a conventional electric motor. These multiple functions can be achieved by applying current on different coils. Fig. 1 shows the schematic of the multifunctional actuator.

When current is applied to the outer coil of the motor part, the induced electromagnetic field would drive the rotor to rotate and then provide active torque. If the current is applied to both the outer and inner coils simultaneously, the MR fluids would produce shear stress under the electromagnetic field induced from the inner coil. As a result, the clutch/brake part could transfer the torque from the motor part to outside as a clutch. By adjusting the current, the actuator could produce controllable torque via the clutch. In another operation, when the current is applied only to the inner coil, the actuator functions as brake. In this situation, with no current applied onto the stator coil, the rotor will not rotate because of the magnetic interaction force between stator and permanent magnets. The advantage of this design is that it compromises with the trade-off between the brake function and bidirectional rotation.

\section{Motion Capture}

Three-dimensional motions of the subject were measured using an optical motion analysis system with 8 cameras (VICON 624, Vicon Motion Systems Ltd, Oxford, United Kingdom) at $120 \mathrm{~Hz}$ (Fig. 4). Before testing, the system was calibrated to ensure the mean residual error was less than 1 $\mathrm{mm}$. The motion capture system was synchronized with a force platform (model OR6-7, AMTI, Watertown, Massachusetts) at a capturing frequency of $120 \mathrm{~Hz}$. Sixteen skin reflective markers ( $9 \mathrm{~mm}$ in diameter) were attached at anatomical landmarks of the lower body according to the Vicon Plug-in Gait model, which included the anterior superior iliac spine, the posterior superior iliac spine, the greater trochanter, the lateral femoral condyle, the tibial tuberosity, the lateral malleolus, the heel and the fifth metatarsal on both right and left sides (Fig. 3). All the markers were attached by the same tester.

\section{Protocol}

The subject was instructed to walk along a straight eight meter path without the assistive knee brace and while wearing the knee brace. The subject walked at a self-selected pace and was given a familiarization period with the knee brace before data was collected. In order to perform inverse dynamics to calculate the lower limb joint kinetics (i.e. joint moments, joint force), only trials in which the right leg of the subject completely landed on the force platform were used. To ensure that the subject walked naturally, the location of the force platform on the floor was not disclosed to the subject. Instead, the subject was instructed to begin walking at different starting positions.

\section{E. Data Analysis}

\section{1) Spatial and Temporal Gait Parameters}

Three successful trials from the normal walking condition and three successful trials from walking with the knee brace were used. The spatial and temporal gait parameters were analyzed according to Vaughan et al (1992) [11]. In order to compare the two walking trials, the data were time normalized

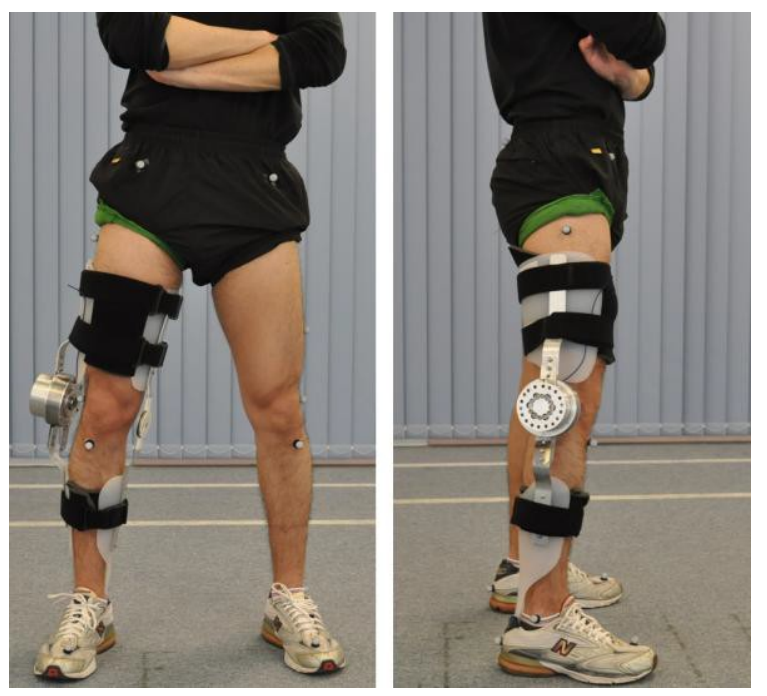

Fig. 3 Integration of knee brace to the user and placement of reflective markers.

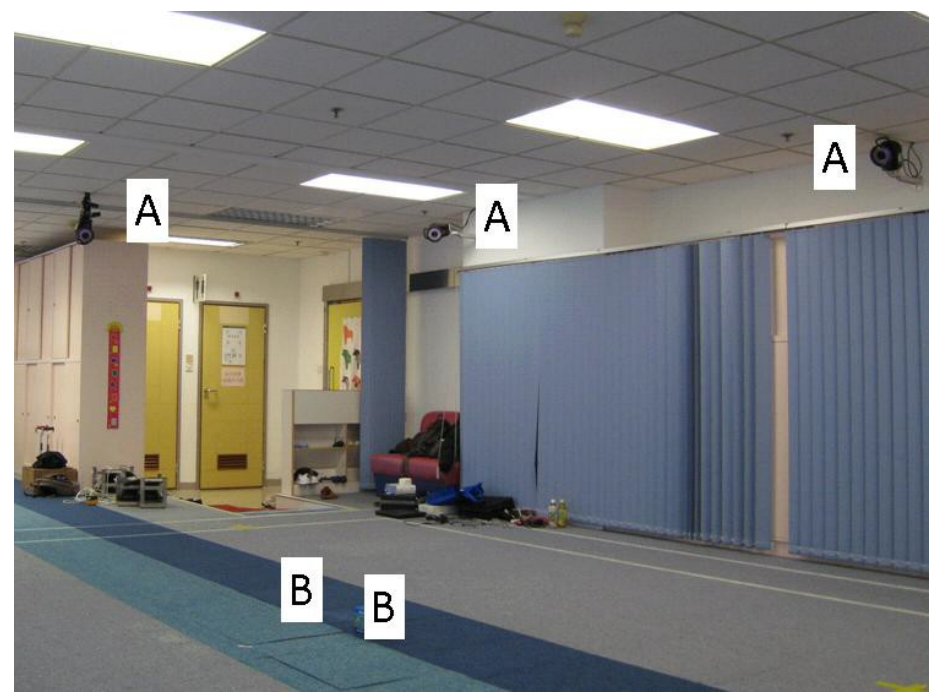

Fig. 4 Experimental setup (A - Infra-red cameras; B - Embedded force platforms). 
to $100 \%$ of the gait cycle. Heel strike and toe off were determined with a $20 \mathrm{~N}$ threshold vertical force. Stride length was determined using the left and right toe markers between successful heel-strikes. Cadence was calculated by dividing the total steps taken by the total time. The average walking speeds in the two trials were calculated by dividing the total distance travelled by the time.

\section{2) Joint Kinematics and Kinetics}

Joint kinematics were analysed for the entire gait cycle whereas joint kinetics were only analysed for the stance phase. The data were analysed using the Vicon Plug-in Gait model and were Butterworth filtered at a cut-off frequency of $10 \mathrm{~Hz}$. Average curves were formed for the vertical ground reaction force (vGRF), joint angle and joint moment profiles between normal walking and walking with the knee brace. Maximum vGRF and maximum joint ranges of motion (ROM) were also determined for comparison.

\section{F. Statistical Analysis}

The differences between maximum vGRF, joint ranges of motions and joint moment between normal walking and walking with the knee brace were analysed using paired t-tests. The level of significance was set at $\mathrm{p} \leq 0.05$.

\section{RESULTS}

\section{A. Spatial and Temporal Gait Parameters}

No significant differences were found for the spatial and temporal gait parameters (Table II), which indicated that the subject's locomotion was not significantly affected by wearing the knee brace.

\section{B. Kinematic Parameters}

The overall patterns of the averaged joint kinematic curves for the two conditions are shown in Fig. 5. Similar movement patterns were found for the hip, knee and ankle joints. It should be noted that the hip was consistently more externally rotated throughout the gait cycle. There was also significantly less knee flexion range of motion (10 degrees, $\mathrm{p}=$ 0.05) when walking with the knee brace (Table III). In addition, significantly greater knee internal rotation and less knee external rotation were observed when walking with the knee brace. However, the differences were only 1 degree and 2 degrees, respectively.

TABLE II

SPATIAL-TEMPORAL GAIT PARAMETERS

\begin{tabular}{lcc}
\hline & Normal Walking & Knee Brace \\
\hline Cycle time (s) & $1.34(0.06)$ & $1.27(0.03)$ \\
Cadence (steps/min) & $88.90(5.28)$ & $94.53(2.00)$ \\
Stride length (m) & $1.46(0.05)$ & $1.45(0.02)$ \\
- Average speed (m/s) & $1.12(0.04)$ & $1.19(0.04)$ \\
\hline Data are presented as mean (SD)
\end{tabular}

\section{Kinetic Parameters}

No significant differences were found for any joint moments at $\mathrm{p} \leq 0.05$ (Table IV). Quantitatively, the mean differences were small, with the greatest mean difference found for the hip extension moment at $1.07 \mathrm{Nm} / \mathrm{kg}$. Similarly, no significant differences were found for the maximum vertical ground reaction force $(\mathrm{p}=0.417)$.

\section{DISCUSSIONS}

The current study evaluated the kinematic and kinetic changes of walking with the assistive knee brace in the "off" mode. This evaluation was important since the current

TABLE III

KINEMATIC DIFFERENCES BETWEEN NORMAL WALKING AND WALKING WITH KNEE BRACE

\begin{tabular}{|c|c|c|c|}
\hline & Mean difference & S.D. & p-value \\
\hline Hip flexion (deg) & 4.59 & $\overline{0.89}$ & 0.09 \\
\hline Hip extension (deg) & 0.77 & 1.75 & 0.64 \\
\hline Hip abduction (deg) & 0.20 & 1.89 & 0.90 \\
\hline Hip adduction (deg) & 0.01 & 0.74 & 0.98 \\
\hline Hip external rotation (deg) & -7.24 & 1.77 & 0.11 \\
\hline Hip internal rotation (deg) & -5.83 & 5.65 & 0.38 \\
\hline Knee flexion (deg) & -10.04 & 1.15 & $0.05 *$ \\
\hline Knee extension (deg) & -1.59 & 0.58 & 0.16 \\
\hline Knee valgus (deg) & -1.62 & 4.59 & 0.71 \\
\hline Knee varus (deg) & -1.59 & 0.66 & 0.18 \\
\hline Knee external rotation (deg) & -0.90 & 0.02 & $0.02 *$ \\
\hline Knee internal rotation (deg) & 1.80 & 0.05 & $0.01 *$ \\
\hline Ankle plantar flexion (deg) & 15.33 & 7.25 & 0.21 \\
\hline Ankle dorsi flexion (deg) & -4.91 & 6.59 & 0.48 \\
\hline Ankle inversion (deg) & 0.16 & 3.82 & 0.96 \\
\hline Ankle eversion (deg) & 1.90 & 7.55 & 0.78 \\
\hline Ankle external rotation (deg) & -0.47 & 1.94 & 0.79 \\
\hline Ankle internal rotation (deg) & 0.03 & 0.22 & 0.89 \\
\hline
\end{tabular}

* Significantly different from normal walking with $\mathrm{p} \leq 0.05$

TABLE IV

KINETIC DIFFERENCES BETWEEN NORMAL WALKING AND WALKING WITH KNEE BRACE

\begin{tabular}{lccc}
\hline & Mean difference & S.D. & p-value \\
\hline vertical GRF max (\%BW) & 4.01 & 4.36 & 0.42 \\
Hip flex mom (Nm/kg) & 0.01 & 0.48 & 0.99 \\
Hip ext mom (Nm/kg) & -1.07 & 1.41 & 0.48 \\
Hip abd mom (Nm/kg) & 0.61 & 0.47 & 0.32 \\
Hip add mom (Nm/kg) & 0.03 & 0.07 & 0.68 \\
Hip ext rot mom (Nm/kg) & 0.00 & 0.01 & 0.53 \\
Hip int rot mom (Nm/kg) & -0.01 & 0.01 & 0.46 \\
Knee flex mom (Nm/kg) & -0.27 & 0.28 & 0.41 \\
Knee ext mom (Nm/kg) & 0.01 & 0.01 & 0.58 \\
Knee varus mom $(\mathrm{Nm} / \mathrm{kg})$ & 0.22 & 0.12 & 0.23 \\
Knee valgus mom $(\mathrm{Nm} / \mathrm{kg})$ & 0.32 & 0.74 & 0.65 \\
Knee int rot mom $(\mathrm{Nm} / \mathrm{kg})$ & -0.02 & 0.02 & 0.38 \\
Knee ext rot mom $(\mathrm{Nm} / \mathrm{kg})$ & 0.12 & 0.11 & 0.36 \\
Ankle dorsiflex mom $(\mathrm{Nm} / \mathrm{kg})$ & 0.02 & 0.03 & 0.50 \\
Ankle plantarflex mom $(\mathrm{Nm} / \mathrm{kg})$ & 0.18 & 0.08 & 0.19 \\
Ankle inversion mom $(\mathrm{Nm} / \mathrm{kg})$ & 0.00 & 0.01 & 0.71 \\
Ankle eversion mom $(\mathrm{Nm} / \mathrm{kg})$ & -0.08 & 0.05 & 0.26 \\
Ankle rot mom $(\mathrm{Nm} / \mathrm{kg})$ & 0.07 & 0.04 & 0.24 \\
Ankle rot mom $(\mathrm{Nm} / \mathrm{kg})$ & -0.16 & 0.04 & 0.12
\end{tabular}





Fig. 5 Joint kinematic profiles of normal walking (grey line) and walking with the knee brace (black line).

knee brace would provide a single DOF assistive torque to the knee joint. As the human knee joint has motions in three planes (flexion/extension, valgus/varus, internal/external rotation), this single DOF assistive torque should only be provided when motions at the other two planes are at a minimum. In order to identify the specific time in which the assistive torque should be provided, it is important to firstly identify changes in the gait pattern when walking with the knee brace. Any changes or hindrance caused by the knee brace should be identified before torque is applied to the knee joint.

Fig. 6 demonstrates the main gait cycle events between the two walking conditions. In general, our findings demonstrated little changes in the spatial and temporal gait parameters, the joint kinematics, and the joint kinetics for the wearer with the knee brace when compared with normal walking, except for the knee flexion angle and the knee external and internal rotation angles (Table III and Table IV). The observed changes in the knee flexion and knee rotation angles could be attributed to the weight and friction caused by the multifunctional actuator. With an added weight to the knee joint, the inertia of the swing leg increases. To account for this added inertia, the subject compensated by adding secondary motion at the transverse plane. As shown in Fig. 5, the hip was consistently more externally rotated throughout the entire gait cycle, indicating that as the subject swung the limb forward, the leg lagged behind in comparison to normal walking. This motion is similar to the hip circumduction strategy often seen in prosthesis users during obstacle clearance [12]. By rotating at the hip joint, this enabled the subject to achieve greater toe clearance even with less knee flexion as compared with normal walking. Since active torque could be provided by our assistive knee brace, some of these changes caused by the friction within the actuator could be attenuated.

We integrated the multifunctional actuator into a custom made knee-ankle-foot orthosis. There were several advantages with the use of a KAFO. First, the KAFO was designed according to the subject's anthropometric measurements. This allowed firm attachment to the subject's lower limb and greatly reduced the amount of shifting that occurred as the leg was in motion. Secondly, the KAFO was able to partially support some of the weight of the multifunctional actuator. In the KAFO, the lower leg segment is attached with the foot segment. Thus when the subject was in stance phase, some of the weight of the actuator is transferred to the ground. However, with this design, the ankle range of motion was also compromised, as evident by the 15 degrees decreased in plantar flexion motion (Table III). Nonetheless, this decrease in ankle range of motion did not transfer to the ankle joint moment. This could be due to the mechanical structure of the orthosis, which produced an assistive plantar flexion moment as the orthosis restored to its original shape after it was bent.

Finally, when the multifunctional actuator was integrated 


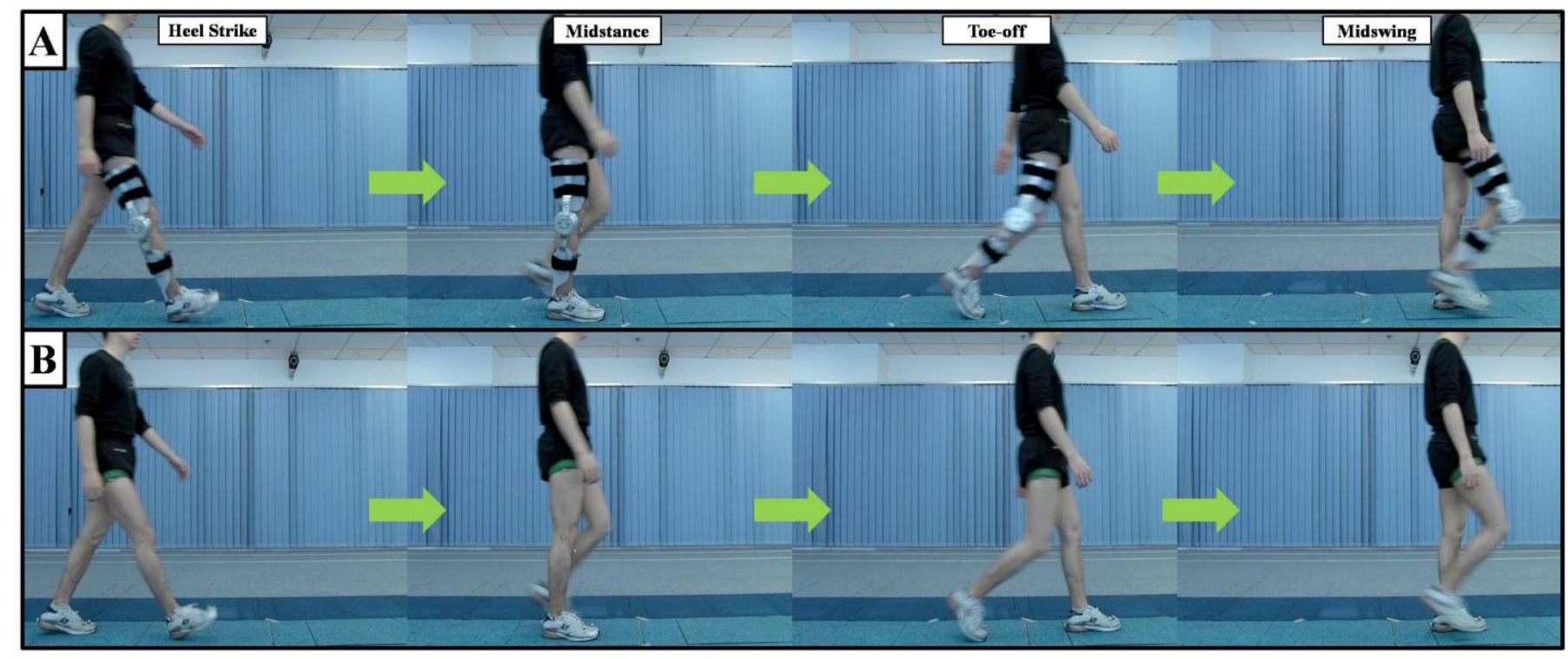

Fig. 6 Gait cycle events for the two walking conditions (A - with the knee brace; B - normal walking).

to the KAFO, it was aligned to the subject's knee joint center. As previously mentioned, the assistive knee brace provided a single DOF torque, whereas the knee rotates in three planes of motion. It was important that the actuator did not interfere with movements in the other two planes. During the design of the assistive knee brace, careful considerations and repeated testing were performed to ensure that motion of the actuator was confined in the sagittal plane. However, it was still not possible to replicate the complex human joint motion with only a single DOF joint. Therefore, it should be noted that the aim during the design of the assistive knee brace was to achieve as minimal resistance to the knee joint as possible, rather than to replicate normal motion.

\section{CONCLUSION}

The current study experimentally investigated the interaction between a user and a knee brace during walking. The assistive knee brace was developed by integrating a multifunctional actuator with a custom-made knee-ankle-foot orthosis in order to minimize excessive shifting and to improve alignment to the knee joint. A comprehensive gait analysis was performed to evaluate changes in the walking gait caused by the developed knee brace. Our evaluation showed that in general, the subject walked with similar gait kinematics, kinetics, and spatial and temporal parameters as compared to normal walking without the knee brace. These results indicated that the knee brace without actuation provided minimal hindrance to the user and assured that assistive torque can be applied to the knee joint while controlling the actuator.

\section{ACKNOWLEDGMENTS}

The work described in this paper was supported by a grant from the Innovation and Technology Commission of the Hong Kong Special Administrative Region, China (Project No. ITS/308/09).

\section{REFERENCES}

[1] J. E. Pratt, B. T. Krupp, C. J. Morse, and S. H. Collins, "The RoboKnee: an exoskeleton for enhancing strength and endurance during walking," Proceedings of the IEEE International Conference on Robotics and Automation, pp. 2430 - 2435, April 2004.

[2] H. Kawamoto and Y. Sankai, "Comfortable power assist control method for walking aid by HAL-3,” IEEE International Conference on Systems, Man and Cybernetics, vol. 4, pp. 190-193, 2002.

[3] Y. Hirata, T. Iwano, and K. Kosuge, "Control of wearable walking helper on slope based on integration of acceleration and GRF information," Proceedings of IEEE/RSJ International Conference on Intelligent Robots and Systems, pp. 3731-3736, 2008.

[4] J. Nikitczuk, B. Weinberg, and C. Mavroidis, "Rehabilitative knee orthosis driven by electro-rheological fluid based actuators," Proceedings of the 2005 IEEE International Conference on Robotics and Automation, pp. 2283-2289, 2005.

[5] F. Ahmadkhanlou, J. Zite, and G. Washington, "A magnetorheological fluid-based controllable active knee brace,” Proceedings of SPIE, vol. 6527, pp. 652700, 2007.

[6] A. Zoss and H. Kazerooni, "Design of an electrically actuated lower extremity exoskeleton,” Advanced Robotics, vol. 20, no. 9, pp. 967-988, 2006.

[7] J. Z. Chen and W. H. Liao, "Design, testing and control of a magnetorheological actuator for assistive knee braces," Smart Materials and Structures, vol. 19, 035029, 2010, doi:10.1088/09641726/19/3/035029.

[8] H. T. Guo and W. H. Liao, "Integrated design and analysis of smart actuators for hybrid assistive knee braces," Proceedings of SPIE Conference on Smart Structures and Materials: Active and Passive Smart Structures and Integrated Systems, vol. 7288, 2009, 72881U1-11, doi: $10.1117 / 12.815964$.

[9] H. T. Guo and W. H. Liao, "Design and control of multifunctional magnetorheological actuators for assistive knee braces," Proceedings of SPIE Conference on Smart Structures and Materials: Active and Passive Smart Structures and Integrated Systems, vol. 7643, 2010, 764310, doi: $10.1117 / 12.847530$.

[10] W. S. Khan, R. K. Jones, L. Nokes, and D. S. Johnson, "How accurate are lockable orthotic knee braces? An objective gait analysis study,” The Knee, vol. 14, pp. 497-499, 2007.

[11] C. L. Vaughan, B. L. Davis, and J. C. O'Connor, "Dynamics of Human Gait," Human Kinetics Publishers Champaign, 1999.

[12] A. H. Vrieling, H. G. van Keeken, T. Schoppen, E. Otten, J. P. K. Halbertsma, A. L. Hof, and K. Postema, "Obstacle crossing in lower limb amputees,” Gait and Posture, vol. 26, pp. 587-594, 2007. 